\title{
KINEMATIC CONTROL OF CONSTRAINED ROBOTIC SYSTEMS
}

\author{
Gustavo M. Freitas* \\ gfreitas@coep.ufrj.br
}

\author{
Antonio C. Leite* \\ toni@coep.ufrj.br
}

\author{
Fernando Lizarralde* \\ fernando@coep.ufrj.br \\ *Department of Electrical Engineering - COPPE \\ Federal University of Rio de Janeiro \\ Rio de Janeiro, RJ, Brazil
}

\section{RESUMO}

Controle de Sistemas Robóticos com Restrições Cinemáticas

Este artigo considera o problema de controle de postura para sistemas robóticos com restrições cinemáticas. A ideia principal é considerar as restrições cinemáticas dos mecanismos a partir de suas equações estruturais, ao invés de usar explicitamente a equação de restrição. Um estudo de caso para robôs paralelos e robôs cooperativos é discutido baseado nos conceitos de cinemática direta, cinemática diferencial, singularidades e controle cinemático. Resultados de simulação, obtidos a partir de um mecanismo Four-Bar linkage, uma plataforma de Gough-Stewart planar e dois robôs cooperativos, ilustram a aplicabilidade e versatilidade da metodologia proposta.

PALAVRAS-CHAVE: robôs paralelos, robôs redundantes, coordenação multi-rôbos, singularidades cinemáticas .

\section{ABSTRACT}

This paper addresses the posture control problem for robotic systems subject to kinematic constraints. The key idea is to consider the kinematic constraints of the mechanisms from their structure equations, instead of explicitly using the constraint equations. A case study

Artigo submetido em 10/03/2011 (Id.: 01293)

Revisado em 27/05/201

Aceito sob recomendação do Editor Associado Prof. Carlos Roberto Minussi for parallel robots and cooperating redundant robots is discussed based on the following concepts: forward kinematics, differential kinematics, singularities and kinematic control. Simulations results, obtained with a Four-Bar linkage mechanism, a planar Gough-Stewart platform and two cooperating robots, illustrate the applicability and versatility of the proposed methodology.

KEYWORDS: Parallel robots, redundant robots, multirobot coordination, kinematic singularities.

\section{INTRODUCTION}

In advanced robotic systems, accuracy, repeatability and load capacity are fundamental skills for carrying out several practical tasks, where the robot end-effector has to perform some operation on a constraint surface or to manipulate an object of the environment (Siciliano et al., 2008). The structure of a robot manipulator consists of a set of rigid bodies or links connected by means of revolute or prismatic joints, integrating a kinematic chain. In general, one end of the chain is fixed to a base, whereas an end-effector is connected to the other end. From a topological point of view, a kinematic chain can be classified in $(i)$ open or serial, when there is only one sequence of links and joints connecting the two ends of the chain, and ( $i i$ ) closed or parallel, when a sequence of links and joints are arranged such that at least one loop exists. Usually, serial chain robots can present limitations in their reachable workspace, kinematic singularities, reduced accuracy and rigidity, or be sensitive to 
scaling. These drawbacks could be overcome by the use of parallel robots to increase rigidity, redundant robots or mobile manipulators (manipulators mounted on a mobile robot, e.g., remotely operated vehicles, wheeled robots) to augment the system workspace and/or avoid singularities in order to accomplish the task of interest (Murray et al., 1994).

Parallel robots provide a rigid connection between the base structure and the payload to be handled by the end-effector, with positioning accuracy superior to the one obtained by serial chain manipulators (Merlet, 1993; Merlet and Gosselin, 2008). The main disadvantages of using parallel robots are: workspace limitation, more complex forward kinematics maps and more involved singularity analysis (Wen and O'Brien, 2003; O'Brien et al., 2006; Simas et al., 2009). For instance, in contrast to serial chain manipulators, the singularities in parallel mechanisms may have different characteristics.

In this context, the singularities can be classified into three basic types (Gosselin and Angeles, 1990; Liu et al., 2003):

(i) configuration space singularities: when the rank of the structure equations drops and, thus, the endeffector loses the ability to move instantaneously in some directions;

(ii) end-effector singularities: when the end-effector loses degrees of freedom (DOF), that is, the motion of the active joints can result in no motion of the end-effector;

(iii) actuator singularities: when the actuator of the manipulator or the active joints cannot produce end-effector forces and torques in some directions.

The kinematic and dynamic control problems for parallel robots are considered in (Kövecses et al., 2003; Cheng et al., 2003; Rosario et al., 2007) and an autonomous control approach to reach the dynamic limits of parallel mechanisms is presented in (Pietsch et al., 2005).

Redundant manipulators have more degrees of freedom ${ }^{1}$ that those strictly necessary to perform a given task. Redundancy is therefore a relative concept for a robot manipulator, depending on the particular type of task to be executed. For example, six DOF are necessary for positioning and orienting a robot end-effector, thus a 6 DOF manipulator is considered non-redundant. However, if only the positioning task is of concern, the same arm becomes redundant. The extra degrees of freedom provide more dexterity to the robot structure, and it can be used to avoid kinematic singularities and collision with obstacles, as well as to optimize the robot motion with respect to a cost function (e.g., joint torque energy). Furthermore, considering the presence of mechanical joint limits, redundant manipulators can also be used to increase the robot workspace (Siciliano, 1990; Chiaverini et al., 2008). In general, multiple cooperating robot arms and mobile manipulators, for instance, belong to the class of redundant robots (Caccavale and Uchiyama, 2008).

The coordination of multiple robots is an essential activity in several industrial applications, such as assembly and manufacturing tasks, where multiple robotic arms are often grasping an object in contact with the environment. Typical examples include: deburring, contour following, grinding, machining, painting, polishing and object aligning (Namvar and Aghili, 2005) or even robot dexterous hands (Caurin and Pedro, 2009). In this framework, a study of the differential kinematics and manipulability indexes for multiple cooperating robot arms with unactuated joints is presented in (Wen and Wilfinger, 1999; Bicchi and Prattichizzo, 2000). Advanced motion and force control of cooperative robotics manipulators with passive joint are considered in (Tinos et al., 2006; Pazelli et al., 2011). A screw-based systematic method to derive the relative Jacobian for two cooperating robots is developed in the recently published work (Ribeiro et al., 2008; Simas et al., 2009).

Motivated by several applications to parallel robots and redundant manipulators, this paper provides a control methodology for robotic systems under kinematic constraints based on a novel proposed method (Wen and O'Brien, 2003). The key idea is to consider the kinematic constraints of these mechanisms from their structure equations, rather than explicitly using the constraint equations. The major advantage of this methodology is its applicability and versatility when different robotic systems are considered. Simulation results are obtained from the kinematic models of a Four-Bar linkage mechanism, a planar Gough-Stewart platform and two cooperating robots.

\section{Terminology and Notation}

In this work, the following notation, definition and assumptions will be adopted:

- $\bar{E}_{a}=\left[\begin{array}{lll}\vec{x}_{a} & \vec{y}_{a} & \vec{z}_{a}\end{array}\right]$ denotes the orthonormal frame $a$ and $\vec{x}_{a}, \vec{y}_{a}, \vec{z}_{a}$ denote the unit vectors of the frame axes.

\footnotetext{
${ }^{1}$ For some authors, this term has the same meaning as the degrees of mobility.
} 
- For a given vector $x \in \mathbb{R}^{n}$, its elements are denoted by $x_{i}$ for $i=1 \cdots n$, that is, $x=\left[\begin{array}{llll}x_{1} & x_{2} & \cdots & x_{n}\end{array}\right]^{T}$.

- $n_{e}$ : number of effective degrees of freedom of the mechanism $^{2}$.

- $n_{t}$ : number of degrees of freedom required to perform a task.

Definition 1 A robotic system can be classified as: (i) non-redundant: $n_{e} \leq n_{t}$; (ii) redundant: $n_{e}>n_{t}$.

Assumption 1 For an open-chain mechanism constituted by $i+1$ links connected by $n$ joints, where the link 0 is fixed, each joint provides a single degree of mobility to the mechanism structure, and $n=i$.

Assumption 2 For a closed-chain mechanism constituted by $i+1$ links, the number of joints $n$ must be greater than $i$. In particular, the number of closed loops is equal to $n-i$.

\section{CONSTRAINED ROBOTIC SYSTEMS}

This section considers the kinematics of the closed-chain robotic systems subject to kinematic constraints on velocity. The general methodology to derive the forward kinematics and the differential kinematics equations for constraint-based robotics systems is to open the loop of the mechanism, propagate the kinematics along the branches and add the kinematic constraints.

Let $p \in(R)^{3}$ be the position of the end-effector frame $\bar{E}_{E}$ with respect to the robot base frame $\bar{E}_{0}$, and $R \in$ $S O(3)$ the orientation of the end-effector frame $\bar{E}_{E}$ with respect to the robot base frame $\bar{E}_{0}{ }^{3}$. In this context, the posture (position and orientation) of the robot endeffector can be obtained from the forward kinematics map as

$$
\{p, R\}=k(\theta),
$$

where $k(\cdot): \mathbb{R}^{n} \mapsto\left\{\mathbb{R}^{3}, S O(3)\right\}$ is a non-linear mapping and $\theta \in \mathbb{R}^{n}$ is the vector of joint variables (or generalized coordinates) expressed in the unconstrained configuration space $\mathcal{Q}$. The vector of active joints (or actuated joints) is denoted by $\theta_{a} \in \mathbb{R}^{n_{a}}$, whereas the vector of passive joints (or unactuated joints) is denoted by $\theta_{p} \in \mathbb{R}^{n_{p}}$, where $n=n_{a}+n_{p}$. Then, we can rearrange the vector of joint angles, such that, $\theta^{T}=\left[\begin{array}{ll}\theta_{a}^{T} & \theta_{p}^{T}\end{array}\right]$. The kinematic

\footnotetext{
${ }^{2}$ The effective degrees of freedom for a mechanism can be calculate by means of the Gruebler's formula, provided that the constraints imposed by the joints are independent (Murray et al., 1994).

${ }^{3}$ Special Orthogonal Group $S O(3)=\left\{R \in \mathbb{R}^{3 \times 3}: R^{T} R=\right.$ $I, \operatorname{det}(R)=1\}$
}

constraints can be locally represented as algebraic constraints in the configuration space $\mathcal{Q}$ by

$$
c(\theta)=0
$$

where $c(\cdot): \mathbb{R}^{n} \mapsto \mathbb{R}^{r}$. Then, the mechanism has $n_{e}=n-r$ effective degrees of freedom and the following assumption is considered:

Assumption 3 The number of active joints is equal to the effective degrees of freedom, that is, $n_{a}=n_{e}$.

Note that, the constraint described in (2) is an example of holonomic constraint. In general, a constraint is said to be holonomic if it restricts the motion of the system to a smooth hypersurface in the configuration space $\mathcal{Q}$ (Murray et al., 1994).

Now, considering that the constraint can be written in terms of the joints velocity vectors, we have

$$
J_{c}(\theta) \dot{\theta}=0
$$

where $J_{c}=\frac{\partial c(\theta)}{\partial \theta} \in \mathbb{R}^{r \times n}$ is named the constraint Jacobian.

On the other hand, the end-effector velocity $v$, composed by the linear velocity $\dot{p}$ and the angular velocity $\omega$ (defined as $\dot{R}=\omega \times R$ (Murray et al., 1994)), can be related with the velocity $\dot{\theta}$ by

$$
v=\left[\begin{array}{c}
\dot{p} \\
\omega
\end{array}\right]=J_{m}(\theta) \dot{\theta},
$$

where $J_{m} \in \mathbb{R}^{n \times n}$ is the manipulator geometric Jacobian (Siciliano et al., 2008).

Partition the Jacobians $J_{c}$ and $J_{m}$ according to the dimension of active-passive joint variables, $\theta_{a}$ and $\theta_{p}$, we have $J_{c}=\left[\begin{array}{ll}J_{c a} & J_{c p}\end{array}\right]$ and $J_{m}=\left[\begin{array}{ll}J_{m a} & J_{m p}\end{array}\right]$. Then, without loss of generality, the equations (3) and (4) can be rewritten as (Wen and O'Brien, 2003; O'Brien et al., 2006):

$$
\begin{aligned}
& 0=J_{c a} \dot{\theta}_{a}+J_{c p} \dot{\theta}_{p}, \\
& v=J_{m a} \dot{\theta}_{a}+J_{m p} \dot{\theta}_{p},
\end{aligned}
$$

where $J_{c a} \in \mathbb{R}^{n_{p} \times n_{a}}, J_{c p} \in \mathbb{R}^{n_{p} \times n_{p}}, J_{m a} \in \mathbb{R}^{m \times n_{a}}$ and $J_{m p} \in \mathbb{R}^{m \times n_{p}}$.

From (5), $\dot{\theta}_{p}$ can be calculated in terms of the active joints as

$$
\dot{\theta}_{p}=-J_{c p}^{-1} J_{c a} \dot{\theta}_{a}
$$

where $J_{c p}$ is invertible if Assumption 3 holds, i.e. the number of passive joints is equal to the number of constraints, $n_{p}=r$. 
Substituting (7) into (6), the differential kinematics equation can be rewritten as

$$
v=\underbrace{\left(J_{m a}-J_{m p} J_{c p}^{-1} J_{c a}\right)}_{\bar{J}\left(\theta_{a}, \theta_{p}\right)} \dot{\theta}_{a},
$$

where $\bar{J} \in \mathbb{R}^{6 \times n-r}$. In the case that $\bar{J}$ be invertible, the control law is similar to the kinematic control case of the serial chain manipulator. For example, for a given desired position $p_{d}(t)$, a proportional control plus feedforward could be proposed:

$$
u=\dot{\theta}_{a}=\bar{J}_{p}\left(\theta_{a}\right)^{\dagger}\left[K\left(p_{d}-p\right)+\dot{p}_{d}\right]
$$

where $K>0$ is the controller gain matrix and $\bar{J}_{p} \in$ $\mathbb{R}^{3 \times n-r}$ is the partition of $\bar{J}$ relating the contribution of the active joint velocity $\dot{\theta}_{a}$ to the end-effector linear velocity $\dot{p}$. Note that a PI controller could also be used, however this controller only guarantees zero steady state error for step-type $p_{d}$. For the orientation control, a representation of the end-effector orientation $R \in S O(3)$ should be considered, e.g. unit quaternion (Murray et al., 1994).

From the analysis of the equation (8), we conclude that a singularity in $\bar{J}$ occurs when the rank of matrix $J_{c p}$ drops. In this case, implies that there is internal motion of the joints, even when the active joints are locked. This type of singularity is named by unstable singularity or actuator singularity (see (Wen and O'Brien, 2003; Kim et al., 2001) for a detailed discussion).

\section{PARALLEL MANIPULATORS}

In this section, a planar Four-Bar linkage mechanism is used to illustrate the posture control problem for parallel manipulators. In sequence, the presented methodology is applied to a planar Gough-Stewart platform. A parallel manipulator is a closed-chain mechanism with end-effector and fixed base, composed by the union of two open kinematic chains at least. Parallel manipulators can present advantages over open-chain manipulators in terms of $(i)$ rigidity of the mechanism, due to the presence of two or more closed chains, and ( $i i)$ actuators allocation, since in general only $n_{e}$ actuators are necessary (Merlet and Gosselin, 2008).

\subsection{Kinematic singularities}

The singularities of the parallel mechanisms can be classified into $(i)$ serial or end-effector singularity and $(i i)$ parallel or actuator singularity. When both singularities occur simultaneously, they are named structural singularity (Gosselin and Angeles, 1990).
In a serial singular configuration, the joints can have a nonzero velocity while the mechanism is at rest. In this case, the end-effector loses degrees of freedom in the task space. On the other hand, in a parallel singular configuration there exist nonzero velocities of the mechanism for which the joint velocities are null, and in this case the end-effector gains some degrees of freedom in the task space. A parallel singularity is especially important for parallel mechanisms since it corresponds to configurations where the robot loses the controlability. Moreover, excessive forces can occur in the vicinity of singular poses and consequently to lead the breakdown of robot parts.

Finally, it is worth to mention that in some cases, singular configurations can be useful. For instance, high amplification factors between the actuated joint motion and the end-effector motion can be essential for improving the sensitivity along some measurements directions for a parallel robot used as a force sensor, or for accurate positioning devices with very small workspace (Merlet and Gosselin, 2008)

\subsection{Planar Four-Bar linkage mechanism}

The considered Four-Bar linkage mechanism is formed by a single closed kinematic chain, composed by the union of two open chains (Figure 1). The mechanical structure consists of four rigid bodies connected by means of revolute joints, where the active joint is $\theta_{a}=\theta_{1}$ and the passive joints are $\theta_{p}=\left[\begin{array}{lll}\theta_{2} & \theta_{3} & \theta_{4}\end{array}\right]^{T}$. Note that, links $l_{3}$ and $l_{4}$ compose one link.

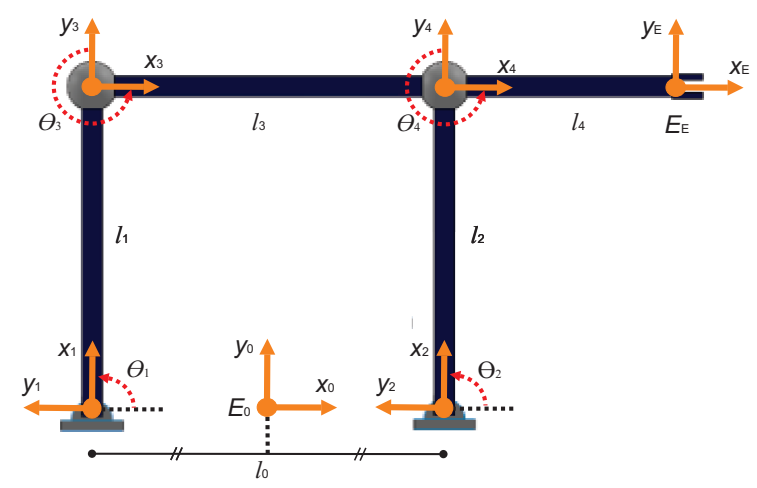

Figure 1: Planar Four-Bar linkage mechanism.

Four-Bar linkages are the simplest, least expensive and most efficient closed-loop kinematic mechanism to perform a wide variety of complicated motions. Linkagetype mechanisms are primarily used for industrial appli- 
cations as machine components and tools, automotive suspensions and bolt cutters.

\subsubsection{Forward kinematics}

The forward kinematics map of a parallel manipulator is described by the posture (position and orientation) of the end-effector frame $\bar{E}_{E}$ with respect to the base frame $\bar{E}_{0}$, derived for each kinematic chain. For parallel mechanisms, the forward kinematics problem is usually much more complex than the inverse kinematics problem, due to the closed loop nature of the mechanism. In order to obtain the forward kinematic, an appropriate frame $\bar{E}_{i}$ for $i=1, \cdots, l$ is attached to $i$-th link. Thus, the structure equations (or loop equations) of the Four-Bar linkage mechanism are given by:

$$
\begin{aligned}
& p=\underbrace{p_{01}+p_{13}+p_{3 E}}_{\text {chain } 1}=\underbrace{p_{02}+p_{24}+p_{4 E}}_{\text {chain } 2}, \\
& \phi=\underbrace{\theta_{1}+\theta_{3}}_{\text {chain 1 }}=\underbrace{\theta_{2}+\theta_{4}}_{\text {chain 2 }},
\end{aligned}
$$

where $\phi \in \mathbb{R}$ represents the end-effector orientation (for the planar case the orientation $R$ is an elementary rotation about the $z$ axis, i.e. $\left.R=R_{z}(\phi)\right)$ and $p_{i j} \in \mathbb{R}^{3}$ is the position vector of the origin of frame $\bar{E}_{j}$ with respect to the origin of the frame $\bar{E}_{i}$. The structure equations of the mechanism introduce constraints between the joint angles of the manipulator. Considering the planar case, where $p \in \mathbb{R}^{2}$ and $\phi \in \mathbb{R}$, equations (10) and (11) correspond to $r=3$ constraints. Thus, the Four-Bar linkage mechanism with $n=4$ joints has $n_{e}=n-r=1$ effective degrees of freedom. This result can also be obtained by using the Gruebler's formula for planar motions (Murray et al., 1994).

Remark 1 For parallel mechanisms, the number of passive joints is always equal to the number of constraints, that is, $n_{p}=r$. Thus, the vector of joint variables $\theta \in \mathbb{R}^{n}$ can be partitioned as $\left(\theta_{a}, \theta_{p}\right)$, where $\theta_{a} \in \mathbb{R}^{n-r}$ are the active joint variables and $\theta_{p} \in \mathbb{R}^{r}$ are the passive joint variables.

The kinematic constraints of the mechanism allow us to control the end-effector orientation by specifying only the angular position of the active joint $\theta_{a}=\theta_{1}$, and the other joint variables must take on values in order to satisfy the structure equations. Thus, the passive joints $\theta_{p} \in \mathbb{R}^{3}$ can be obtained as a function of active joints $\theta_{a} \in \mathbb{R}$ by means of the forward kinematics equation of the mechanism, by using the chain 1

$$
\begin{aligned}
p= & p_{01}+p_{13}+p_{3 E}=-\frac{l_{0}}{2} \vec{x}_{0}+l_{1} R_{01}\left(\theta_{1}\right) \vec{x}_{0}+ \\
& \left(l_{3}+l_{4}\right) R_{01}\left(\theta_{1}\right) R_{13}\left(\theta_{3}\right) \vec{x}_{0}
\end{aligned}
$$

or the chain 2

$$
\begin{aligned}
p= & p_{02}+p_{24}+p_{4 E}=\frac{l_{0}}{2} \vec{x}_{0}+l_{2} R_{02}\left(\theta_{2}\right) \vec{x}_{0}+ \\
& l_{4} R_{02}\left(\theta_{2}\right) R_{24}\left(\theta_{4}\right) \vec{x}_{0}
\end{aligned}
$$

where $R_{i j}\left(\theta_{i}\right) \in S O(3)$ denotes the orientation of the frame $\bar{E}_{j}$ with respect to the frame $\bar{E}_{i}$. Note that, in this case, $R_{i j}$ is an elementary rotation matrix by an angle $\theta_{i}$ about the axis $z$ of the frame $\bar{E}_{i}$. After the use of geometric and algebraic identities, the passive joints $\theta_{p}$ are obtained by

$$
\begin{aligned}
& \theta_{2}=\pi-\arccos \left(\frac{l_{0}^{2}-l_{1}^{2}+l_{d}^{2}}{2 l_{0} l_{d}}\right)-\arccos \left(\frac{l_{2}^{2}-l_{3}^{2}+l_{d}^{2}}{2 l_{2} l_{d}}\right), \\
& \theta_{3}=\pi+\arccos \left(\frac{l_{1}^{2}-l_{0}^{2}+l_{d}^{2}}{2 l_{1} l_{d}}\right)+\arccos \left(\frac{l_{3}^{2}-l_{2}^{2}+l_{d}^{2}}{2 l_{3} l_{d}}\right), \\
& \theta_{4}=2 \pi-\arccos \left(\frac{l_{2}^{2}+l_{3}^{2}-l_{d}^{2}}{2 l_{2} l_{3}}\right),
\end{aligned}
$$

where

$$
l_{d}^{2}=l_{0}^{2}+l_{1}^{2}-2 l_{o} l_{1} \cos \left(\theta_{1}\right)
$$

In the case that $l_{3}=l_{0}$ and $l_{1}=l_{2}$, there always exists solution for $\theta_{3}$ and we has that $\theta_{2}=\theta_{1}$ and $\theta_{3}=\theta_{4}$.

Now, it is possible to obtain the end-effector position from (12) or (13), as well as the end-effector orientation from (11), in terms of the active joint $\theta_{a}=\theta_{1}$.

Remark 2 A more direct technique to solve the inverse kinematics problem and obtain the passive joint variables $\theta_{p}$ is to apply the methods based on PadenKahan subproblems, in particular the subproblems 1 and 3 (Murray et al., 1994), which are geometrically meaningful and numerically stable.

\subsubsection{Differential kinematics}

Analogously, the differential kinematics of a parallel manipulator is computed by considering the various open kinematic chains that compose the mechanism structure. The end-effector velocity $v \in \mathbb{R}^{3}$ can be obtained from the time derivative of structure equations, resulting in a Jacobian matrix for each serial chain

$$
v=S J_{1}\left[\begin{array}{c}
\dot{\theta}_{1} \\
\dot{\theta}_{3}
\end{array}\right]=S J_{2}\left[\begin{array}{c}
\dot{\theta}_{2} \\
\dot{\theta}_{4}
\end{array}\right],
$$


where $S \in \mathbb{R}^{3 \times 6}$ is a selection matrix given by:

$$
S=\left[\begin{array}{llllll}
1 & 0 & 0 & 0 & 0 & 0 \\
0 & 1 & 0 & 0 & 0 & 0 \\
0 & 0 & 0 & 0 & 0 & 1
\end{array}\right],
$$

and the Jacobian matrices $J_{1} \in \mathbb{R}^{6 \times 2}$ and $J_{2} \in \mathbb{R}^{6 \times 2}$ are ${ }^{4}$ :

$$
\begin{aligned}
& J_{1}=\left[\begin{array}{cc}
\vec{z}_{0} \times p_{1 E} & \vec{z}_{0} \times p_{3 E} \\
\vec{z}_{0} & \vec{z}_{0}
\end{array}\right], \\
& J_{2}=\left[\begin{array}{cc}
\vec{z}_{0} \times p_{2 E} & \vec{z}_{0} \times p_{4 E} \\
\vec{z}_{0} & \vec{z}_{0}
\end{array}\right],
\end{aligned}
$$

with

$$
\begin{aligned}
& p_{1 E}=l_{1} R_{01}\left(\theta_{1}\right) \vec{x}_{0}+p_{3 E}, \\
& p_{2 E}=l_{2} R_{02}\left(\theta_{2}\right) \vec{x}_{0}+p_{4 E}, \\
& p_{3 E}=\left(l_{3}+l_{4}\right) R_{01}\left(\theta_{1}\right) R_{13}\left(\theta_{3}\right) \vec{x}_{0}, \\
& p_{4 E}=l_{4} R_{02}\left(\theta_{2}\right) R_{24}\left(\theta_{4}\right) \vec{x}_{0} .
\end{aligned}
$$

The Jacobian of the mechanism can be rewritten in a more usual form, stacking the Jacobian of each open chain:

$$
\underbrace{\left[\begin{array}{cc}
S J_{1} & 0 \\
0 & S J_{2}
\end{array}\right]}_{J} \underbrace{\left[\begin{array}{c}
\dot{\theta_{1}} \\
\dot{\theta_{3}} \\
\dot{\theta_{2}} \\
\dot{\theta_{4}}
\end{array}\right]}_{\dot{\theta}}=\underbrace{\left[\begin{array}{c}
I \\
I
\end{array}\right]}_{A} v,
$$

or equivalently

$$
J \dot{\theta}=A v,
$$

where the matrix $A \in \mathbb{R}^{6 \times 3}$ has full column rank. Using this notation, it is possible obtain the constraint Jacobian $J_{c} \in \mathbb{R}^{3 \times 4}$ and the manipulator Jacobian $J_{m} \in \mathbb{R}^{3 \times 4}$ by means of:

$$
J_{c}=\tilde{A} J, \quad J_{m}=A^{\dagger} J,
$$

where $\widetilde{A} \in \mathbb{R}^{3 \times 6}$ is the annihilator of $A$, such that, $\widetilde{A} A=0$, and $A^{\dagger} \in \mathbb{R}^{3 \times 6}$ is the pseudo-inverse of $A$, such that $A^{\dagger} A=I$. A possible choice is $\widetilde{A}=\left[\begin{array}{ll}I & -I\end{array}\right]$ and $A^{\dagger}=\left[\begin{array}{ll}I & 0\end{array}\right]$. From (8), $\bar{J} \in \mathbb{R}^{3 \times 1}$ can be calculated by $\bar{J}=J_{m a}-J_{m p} J_{c p}^{-1} J_{c a}$.

\subsubsection{Kinematic control}

In this section, the kinematic control approach is used to modify the posture of the Four-Bar linkage mechanism in order to perform a task of interest. Here, we assume that the control objective is to drive the current end-effector orientation $\phi$ to a desired time-varying orientation $\phi_{d}(t)$, that is,

$$
\phi \rightarrow \phi_{d}(t), \quad e_{\phi}=\phi_{d}(t)-\phi \rightarrow 0,
$$

\footnotetext{
${ }^{4} \times$ denotes the vector cross product.
}

where $e_{\phi} \in \mathbb{R}$ is the orientation error.

The control scheme to be designed has to command the velocity of the active joint $\dot{\theta}_{a}=\dot{\theta}_{1}$ in order to achieve the control objective (25). Then, using a proportional control law plus feedforward action

$$
u=\dot{\theta}_{a}=\left(\bar{J}_{3}\right)^{-1}\left(k_{\phi} e_{\phi}+\dot{\phi}_{d}\right),
$$

where $\bar{J}_{3}$ is the third element of $\bar{J}$ and the orientation error dynamics is governed by $\dot{e}_{\phi}+k_{\phi} e_{\phi}=0$, provided that the mechanism motions are away from singular configurations. Hence, by a proper choice of $k_{\phi}$ as a positive constant implies that $\lim _{t \rightarrow \infty} e_{\phi}(t)=0$.

\subsection{Planar Gough-Stewart platform}

Another usual example of parallel mechanisms is the Gough-Stewart platform. Typically, some applications of this structure include: aircraft flight simulators, antennas and telescopes positioning systems, machine tool and crane technologies, and orthopedic surgery. In this section, we consider a planar version of the GoughStewart platform (Figure 2). The mechanical structure is composed by the union of three open chains and has nine joints, where three prismatic joints are active $\theta_{a}=\left[\begin{array}{lll}d_{2} & d_{5} & d_{8}\end{array}\right]^{T}$, and six revolute joints are passive $\theta_{p}=\left[\begin{array}{llllll}\theta_{1} & \theta_{3} & \theta_{4} & \theta_{6} & \theta_{7} & \theta_{9}\end{array}\right]^{T}$. We can obtain the effective degrees of freedom for this mechanism by applying the Gruebler's formula for planar motions (Murray et al., 1994):

$$
n_{e}=3(i-n)+\sum_{j=1}^{n} f_{i}=3,
$$

where $i$ is the number of mobile links in the mechanism, $n$ is the number of joints and $f_{i}$ is the number of degrees of freedom for the $i$-th joint. From (27), we conclude that the mechanism has three effective degrees of freedom, which allow us to control the position and orientation of the platform respectively, in order to perform planar positioning tasks.

\subsubsection{Forward kinematics}

The solution of the forward kinematics problem for a Gough-Stewart platform is a very difficult task due to the large number and complicated form of the constraints. From the length of the links, we can solve the structure equations to find the joint angles and then determine the platform posture. The forward kinematics map of the mechanism can be obtained by calculating the position and orientation of the frame $\bar{E}_{s}$ with respect to the base frame $\bar{E}_{0}$, specified for each kinematic 


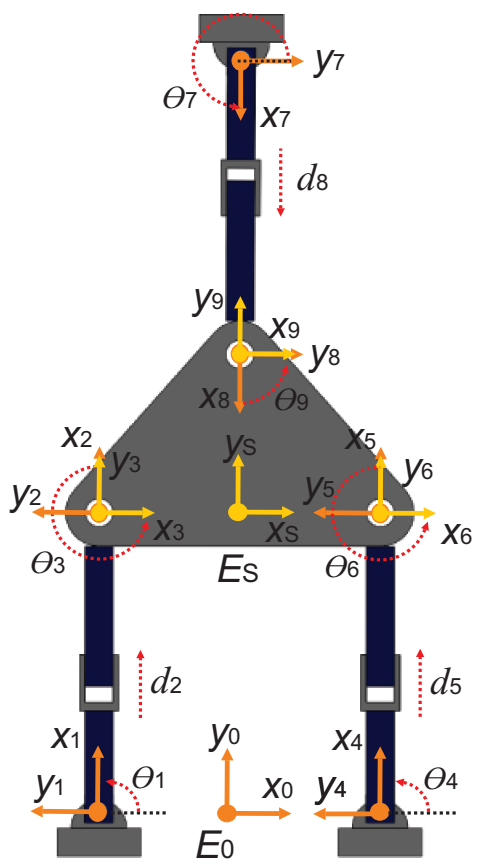

Figure 2: Planar Gough-Stewart platform.

chain:

$$
\begin{aligned}
& p_{s}=\underbrace{p_{01}+p_{13}+p_{3 s}}_{\text {chain } 1}=\underbrace{p_{04}+p_{46}+p_{6 s}}_{\text {chain 2 }}=\underbrace{p_{07}+p_{79}+p_{9 s}}_{\text {chain 3 }}, \\
& \phi_{s}=\underbrace{\theta_{1}+\theta_{3}}_{\text {chain 1 }}=\underbrace{\theta_{4}+\theta_{6}}_{\text {chain 2 }}=\underbrace{\theta_{7}+\theta_{9}}_{\text {chain 3 }},
\end{aligned}
$$

where $\phi_{s} \in \mathbb{R}$ represents the platform orientation (for the planar case the orientation $R$ is an elementary rotation about the $z$ axis, i.e. $\left.R=R_{z}(\phi)\right)$ and $p_{01}, p_{04}$ and $p_{07}$ are assumed to be constant and known, with

$$
\begin{aligned}
& p_{03}=d_{2} R_{01}\left(\theta_{1}\right) \vec{x}_{0}, \\
& p_{3 s}=l R_{01}\left(\theta_{1}\right) R_{13}\left(\theta_{3}\right) \vec{x}_{0}, \\
& p_{0 s}=p_{03}+p_{3 s}, \\
& p_{46}=d_{5} R_{04}\left(\theta_{4}\right) \vec{x}_{0}, \\
& p_{6 s}=l R_{04}\left(\theta_{4}\right) R_{46}\left(\theta_{6}\right) \vec{x}_{0}, \\
& p_{4 s}=p_{46}+p_{6 s}, \\
& p_{79}=d_{8} R_{07}\left(\theta_{7}\right) \vec{x}_{0}, \\
& p_{9 s}=l R_{07}\left(\theta_{7}\right) R_{79}\left(\theta_{9}\right) \vec{x}_{0}, \\
& p_{7 s}=p_{79}+p_{9 s},
\end{aligned}
$$

where $l \in \mathbb{R}$ is the distance from the frame $\bar{E}_{s}$ to frames $\bar{E}_{3}, \bar{E}_{6}$ and $\bar{E}_{9}$ respectively.

Note that, considering a parallel mechanism, the structure equations allow the formulation of a system of equations, containing the kinematic constraint of the mecha- nism, that can be used to calculate the angle of the passive joints $\theta_{p}$ in terms of the angle of the active joints $\theta_{a}$. The solution for this system of equations can be trivial, as in the case of the Four-Bar linkage mechanism, or be complex, for the case of the Gough-Stewart platform presented in this section.

The forward kinematics map of the planar GoughStewart platform can be obtained by using the methodology presented in (Zhang and Gao, 2006), where the system with six equations and six unknowns variables $\left(\theta_{p} \in \mathbb{R}^{6}\right)$ is solved by means of the Ritt-Wu's characteristic set method.

\subsubsection{Differential kinematics}

The differential kinematics equation is obtained considering the various open chains, which compose the mechanism structure. The platform velocity can be derived by differentiating the structure equation, obtaining a Jacobian matrix for each chain:

$$
v=S J_{1}\left[\begin{array}{c}
\dot{\theta}_{1} \\
\dot{d}_{2} \\
\dot{\theta}_{3}
\end{array}\right]=S J_{2}\left[\begin{array}{c}
\dot{\theta}_{4} \\
\dot{d}_{5} \\
\dot{\theta}_{6}
\end{array}\right]=S J_{3}\left[\begin{array}{c}
\dot{\theta}_{7} \\
\dot{d}_{8} \\
\dot{\theta}_{9}
\end{array}\right]
$$

where $v^{T}=\left[\begin{array}{ll}\dot{p}_{s}^{T} & \dot{\phi}_{s}\end{array}\right], S \in \mathbb{R}^{3 \times 6}$ is the selection matrix given in (15), and the Jacobian matrices $J_{1} \in \mathbb{R}^{6 \times 3}, J_{2} \in$ $\mathbb{R}^{6 \times 3}$ and $J_{3} \in \mathbb{R}^{6 \times 3}$ are:

$$
\begin{aligned}
& J_{1}=\left[\begin{array}{ccc}
\vec{z}_{0} \times p_{0 s} & R_{01}\left(\theta_{1}\right) \vec{x}_{0} & \vec{z}_{0} \times p_{3 s} \\
\vec{z}_{0} & 0 & \vec{z}_{0}
\end{array}\right], \\
& J_{2}=\left[\begin{array}{ccc}
\vec{z}_{0} \times p_{4 s} & R_{04}\left(\theta_{4}\right) \vec{x}_{0} & \vec{z}_{0} \times p_{6 s} \\
\vec{z}_{0} & 0 & \vec{z}_{0}
\end{array}\right], \\
& J_{3}=\left[\begin{array}{ccc}
\vec{z}_{0} \times p_{7 s} & R_{07}\left(\theta_{7}\right) \vec{x}_{0} & \vec{z}_{0} \times p_{9 s} \\
\vec{z}_{0} & 0 & \vec{z}_{0}
\end{array}\right] .
\end{aligned}
$$

It is important to note that the Jacobians $J_{1}, J_{2}$ and $J_{3}$ depends on the angles of the active and passive joints. For the planar Gough-Stewart platform, it is not trivial to calculate the position of the passive joints $\theta_{p}$ in terms of the active joints $\theta_{a}$ by using its forward kinematics equations. However, in order to obtain the differential kinematics equations of the system, the position of the passive joints can be obtained, for instance, by integrating the velocity of the passive joints $(7)$, i.e. $\theta_{p}=\int J_{c p}^{-1} J_{c a} \dot{\theta}_{a} d \tau$. 
The Jacobian can be rewritten in a more conventional way, stacking the Jacobians for each open chain.

$$
\underbrace{\left[\begin{array}{ccc}
S J_{1} & 0 & 0 \\
0 & S J_{2} & 0 \\
0 & 0 & S J_{3}
\end{array}\right]}_{J} \underbrace{\left[\begin{array}{c}
\dot{\theta}_{1} \\
\dot{d}_{2} \\
\dot{\theta}_{3} \\
\dot{\theta}_{4} \\
\dot{d}_{5} \\
\dot{\theta}_{6} \\
\dot{\theta}_{7} \\
\dot{d}_{8} \\
\dot{\theta}_{9}
\end{array}\right]}_{\dot{\theta}}=\underbrace{\left[\begin{array}{c}
I \\
I \\
I
\end{array}\right]}_{A} v
$$

or, equivalently

$$
J \dot{\theta}=A v,
$$

where the matrix $A \in \mathbb{R}^{9 \times 3}$ has full column rank. By using this notation, it is possible to obtain the constraint Jacobian $J_{c} \in \mathbb{R}^{6 \times 9}$ and the manipulator Jacobian $J_{m} \in$ $\mathbb{R}^{3 \times 9}$ by means of:

$$
J_{c}=\tilde{A} J, \quad J_{m}=A^{\dagger} J,
$$

where a possible choice for $\tilde{A}$ and $A^{\dagger}$ is given by $\widetilde{A}=$ $\left[\begin{array}{lll}I & -I & 0\end{array}\right]$ and $A^{\dagger}=\left[\begin{array}{lll}I & 0 & 0\end{array}\right]$.

As previously stated, from (8) and by using $J_{c}$ and $J_{m}$, the differential kinematics equation of the mechanism is given by $v=\bar{J} \dot{\theta}_{a}$, where $\bar{J} \in \mathbb{R}^{3 \times 3}$ is given by $\bar{J}=$ $J_{m a}-J_{m p} J_{c p}^{-1} J_{c a}$.

\subsubsection{Kinematic control}

In accordance with the Section 3.2.3, the kinematic control approach can be used again to modify the posture of the platform, in order to perform a planar positioning task. Here, we assume that the control objective is to follow a desired time-varying posture $x_{s d}(t)=\left[\begin{array}{ll}p_{s d}^{T}(t) & \phi_{s d}(t)\end{array}\right]^{T}$ from the current platform posture $x_{s}=\left[\begin{array}{ll}p_{s}^{T} & \phi_{s}\end{array}\right]^{T}$, that is,

$$
x_{s} \rightarrow x_{s d}(t), \quad e_{s}=x_{s d}-x_{s} \rightarrow 0,
$$

where $e_{s} \in \mathbb{R}^{3}$ is the platform posture error.

Now, the control scheme to be designed has to command the velocity of the active joint $\dot{\theta}_{a}=\left[\begin{array}{lll}\dot{d}_{2} & \dot{d}_{5} & \dot{d}_{8}\end{array}\right]^{T}$, in order to achieve the control objective (45). Then, using a control law based on a proportional with feedforward action

$$
u=\dot{\theta}_{a}=\bar{J}^{-1}\left(K_{s} e_{s}+\dot{x}_{s d}\right),
$$

where $K_{s}$ is the controller gain matrix, the posture error dynamics is governed by $\dot{e}_{s}+K_{s} e_{s}=0$, provided that the platform motions are away from singular configurations. Hence, by a proper choice of $K_{s}$ as a positive definite matrix, implies that $\lim _{t \rightarrow \infty} e_{s}(t)=0$.

\section{COOPERATING MANIPULATORS}

In the robotics area, many tasks are difficult or even impossible to be performed by using a single robot. Typical examples include: positioning of heavy payloads, complex assembly of multiple parts or manipulation of flexible objects. These tasks become feasible with the employment of more than one robot operating cooperatively (Caccavale and Uchiyama, 2008). The mechanism presented in Figure 3 is constituted by a closed kinematic chain, where all joints are active, and considers a continuous contact of the end-effector with the manipulated object.

In general, cooperating robots correspond to overactuated systems or redundant systems, where the effective degrees of freedom are higher than those strictly required to perform a given task $\left(n_{e}>n_{t}\right)$. This capacity increases the dexterity of the mechanism, and can be used to avoid joint limits, singularities and workspace obstacles, as well as to minimize the energy consumption and joint torques or to optimize a performance index (e.g., manipulability).

\subsection{Forward kinematics}

The forward kinematics map for a redundant robotic system composed by two cooperating robots can be described by means of the posture of the frame attached to the manipulated object $\bar{E}_{c}$ with respect to the base frame $\bar{E}_{0}$, determined for each manipulator that belongs to the robotic system. The structure equations of the redundant mechanism illustrated in Figure 3 are given respectively by

$$
\begin{aligned}
& p_{c}=\underbrace{p_{01}+p_{1 c}}_{\text {robot } 1}=\underbrace{p_{02}+p_{2 c}}_{\text {robot } 2}, \\
& \phi_{c}=\underbrace{\phi_{01}+\phi_{1 c}}_{\text {robot } 1}=\underbrace{\phi_{02}+\phi_{2 c}}_{\text {robot } 2},
\end{aligned}
$$

where

$p_{0 i} \in \mathbb{R}^{3}:$ is the position vector of the end-effector frame of the $i$-th manipulator $\bar{E}_{i}$ with respect to the base frame $\bar{E}_{0}$.

$p_{i c} \in \mathbb{R}^{3}$ : is the position vector of the manipulated object frame $\bar{E}_{c}$ with respect to the end-effector frame of the $i$-th manipulator $\bar{E}_{i}$.

$\phi_{0 i} \in \mathbb{R}^{3}:$ denotes the orientation of the end-effector frame of the $i$-th manipulator $\bar{E}_{i}$ with respect to the base frame $\bar{E}_{0}$. 


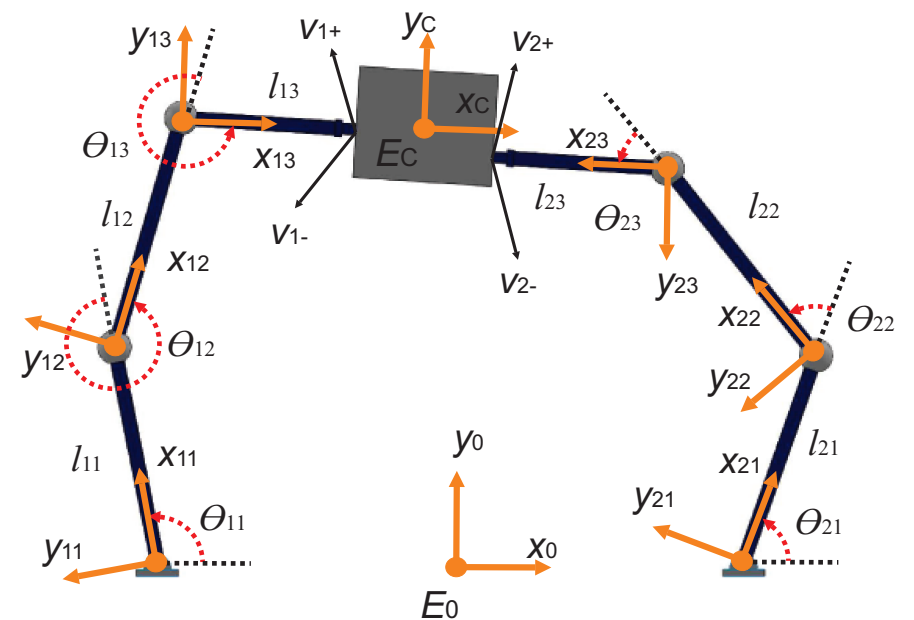

Figure 3: Cooperating robot arms carrying a rigid object.

$\phi_{i c} \in \mathbb{R}^{3}$ : denotes the orientation of the manipulated object frame $\bar{E}_{c}$ with respect to the end-effector frame of the $i$-th manipulator $\bar{E}_{i}$.

The structure equations introduce kinematic constraints on the system, due to the continuous contact of the robots with the manipulated object. In contrast with parallel mechanism, the number of constraints is not equal to the number of passive joints of the robots. Indeed, for the mechanism presented in Figure 3, the passive joints are associated to the contact points between the manipulators and the object (Caccavale and Uchiyama, 2008).

\subsection{Differential kinematics}

Considering the open chain, the end-effector velocity $v_{i}^{+}$ of the $i$-th manipulator is related with the velocities of the joints $\theta_{i}$ by

$$
v_{i}^{+}=J_{i}\left(\theta_{i}\right) \dot{\theta}_{i},
$$

where $J_{i}$ is the Jacobian of the $i$-th manipulator, obtained as a function of the joint angles $\theta_{i}$.

Now, we consider $v_{c}$ the velocity of the frame $\bar{E}_{c}$ fixed on the manipulated object. The object velocity $v_{i}^{-}$at the contact points is related with $v_{c}$ by means of

$$
v_{i}^{-}=A_{i} v_{c}, \quad A_{i}=\left[\begin{array}{cc}
I & -p_{i c} \times \\
0 & I
\end{array}\right]
$$

where $A_{i}$ is the adjoint transformation which relates the velocities of the object frame $\bar{E}_{c}$ and the end-effector frame of the $i$-th manipulator $\bar{E}_{E_{i}}$.
The relative velocity of the each contact point can be parameterized by a velocity vector $w_{i}$ by using:

$$
v_{i}^{-}=v_{i}^{+}+H_{i}^{T} w_{i}
$$

where the columns of the matrix $H_{i}^{T}$ represent the directions for free motion at the contact points.

The Jacobian can be rewritten in a more conventional way stacking the Jacobians for each open kinematic chain as

$$
\underbrace{\left[\begin{array}{cc}
J_{1} & 0 \\
0 & J_{2}
\end{array}\right]}_{J} \dot{\theta}=\underbrace{\left[\begin{array}{c}
v_{1}^{+} \\
v_{2}^{+}
\end{array}\right]}_{v^{+}},
$$

or equivalently

$$
J \dot{\theta}=v^{+} .
$$

Thus, the differential kinematics relations can be rewritten as

$$
v^{+}+H^{T} w=v^{-}, \quad v^{-}=A v_{c},
$$

where $w=\left[\begin{array}{ll}w_{1}^{T} & w_{2}^{T}\end{array}\right]^{T}, H=\left[\begin{array}{ll}H_{1}^{T} & H_{2}^{T}\end{array}\right]^{T}$ and $A^{T}=\left[\begin{array}{ll}A_{1}^{T} & A_{2}^{T}\end{array}\right]$ has full rank.

The definition $\dot{\theta}_{p}=w$ and $\dot{\theta}_{a}=\dot{\theta}$ allow us to represent the system in a more generally form, according to (5) and (6), by means of (Wen and Wilfinger, 1999)

$$
\begin{gathered}
\underbrace{\tilde{A}\left[\begin{array}{ll}
J & H^{T}
\end{array}\right]}_{J_{c}}\left[\begin{array}{c}
\dot{\theta}_{a} \\
\dot{\theta}_{p}
\end{array}\right]=0, \\
\underbrace{A^{\dagger}\left[\begin{array}{ll}
J & H^{T}
\end{array}\right]}_{J_{m}}\left[\begin{array}{c}
\dot{\theta}_{a} \\
\dot{\theta}_{p}
\end{array}\right]=v_{c},
\end{gathered}
$$

where $\widetilde{A}$ is the annihilating matrix such that, $\widetilde{A} A=0$, and $A^{\dagger}$ is the pseudo-inverse of matrix $A$ such 
that $A^{\dagger} A=I$. A possible choice for $\tilde{A}$ and $A^{\dagger}$ is given by $\widetilde{A}=\left[\begin{array}{ll}A_{2} & -A_{1}\end{array}\right]$ and $A^{\dagger}=\left[\begin{array}{ll}A_{1}^{-1} & 0\end{array}\right]$.

Note that, from (8) and by using $J_{c}$ and $J_{m}$, the differential kinematics equation of the object is given by $v_{c}=$ $\bar{J} \dot{\theta}_{a}$, where $\bar{J} \in \mathbb{R}^{3 \times 6}$ is given by $\bar{J}=J_{m a}-J_{m p} J_{c p}^{-1} J_{c a}$.

\subsection{Selection matrices}

The kinematic constraints of the robotic system due to the contact points are properly represented by means of a selection matrix $H$. This matrix acts as a filter that accepts or rejects components of motion at the contact point.

Considering the example presented in Figure 3, for the planar case a multiple robot system with compliant grippers does not allow translational and rotational motions of the manipulated object, which implies that $H=0$. On the other hand, for a multiple robot system without grippers, contact points with friction can be considered. In this case, only angular motions between the end-effector and the object are allowed and the selection matrix $H$ is given by

$$
H=\left[\begin{array}{l}
0 \\
0 \\
1
\end{array}\right] .
$$

Some examples of other types of contacts and associated values of the selection matrix $H$ are presented in (Murray et al., 1994; Wen and Wilfinger, 1999).

\subsection{Kinematic control}

In this section, the kinematic control approach will be used again to modify the posture of the robot manipulators, in order to perform a planar manipulation task with the object of interest. Here, we assume that the control objective is to lead the current posture of the object $x_{c}=\left[\begin{array}{ll}p_{c}^{T} & \phi_{c}\end{array}\right]^{T}$ to a desired time varying posture $x_{c d}(t)=\left[\begin{array}{ll}p_{c d}^{T}(t) & \phi_{c d}(t)\end{array}\right]^{T}$, that is,

$$
x_{c} \rightarrow x_{c d}(t), \quad e_{c}=x_{c d}(t)-x_{c} \rightarrow 0,
$$

where $e_{c}$ is posture error of the object.

The control scheme to be designed has to command the velocity of the all active joints of the multiple robots system $\dot{\theta}_{a}$ in order to achieve the control objective (58). According to the differential kinematics of the actuated mechanism $v_{c}=\bar{J} \dot{\theta}_{a}$ with $n_{e}=n_{t}$, the joint velocities can be obtained from the simple inversion of the Jacobian matrix $\bar{J}$ by using $\dot{\theta}_{a}=\bar{J}^{-1} v$, where $v$ denotes the Cartesian control law, which is properly designed to avoid singular configurations.
On the other hand, for a redundant mechanism such that $n_{e}>n_{t}$, the same relationship can be rewritten in a generic form as (Sciavicco and Siciliano, 2000; Chiaverini et al., 2008):

$$
\dot{\theta}_{a}=\bar{J}^{\dagger} v+\underbrace{\left(I-\bar{J}^{\dagger} \bar{J}\right)}_{\mathcal{P}} \dot{\theta}_{a_{0}},
$$

where $\mathcal{P}$ denotes the orthogonal projection matrix in the null space of $\bar{J}\left(i . e . \bar{J} \mathcal{P}=0\right.$ ) and $\dot{\theta}_{a_{0}}$ is a vector of arbitrary velocities of the active joints. Note that, the right side of (59) can be interpreted as a null space velocity, whose effect is to generate internal motions that reconfigure the robot structure without changing the end-effector posture.

Considering the kinematics control problem of the overactuated mechanism $\left(n_{e}>n_{t}=1\right)$, the control signal is equivalent to the velocity of the active joints, that is, $u=$ $\dot{\theta}_{a}$. Then, using a control law based on a proportional with feedforward action

$$
u=\bar{J}^{\dagger}\left(\dot{x}_{c d}+K_{c} e_{c}\right)+\left(I-\bar{J}^{\dagger} \bar{J}\right) \bar{u},
$$

where $\bar{u}$ is an auxiliary control signal, the posture error dynamics is governed by $\dot{e}_{c}+K_{c} e_{c}=0$, where $K_{c}$ is the controller gain matrix, since the right side of the (60) is projected in the null space of $\bar{J}$. Hence, for a proper choice of $K_{c}$ as a positive definite matrix, implies that $\lim _{t \rightarrow \infty} e_{c}(t)=0$.

The auxiliary control $\bar{u}$ can be also chosen in order to improve the performance of the mechanism for the task execution. A typical choice is

$$
\bar{u}=\bar{K}\left(\frac{\partial f\left(\theta_{a}\right)}{\partial \theta_{a}}\right)^{T},
$$

where $\bar{K}>0$ is a gain factor and $f\left(\theta_{a}\right)$ is an objective function in terms of the active joint variables, that can be chosen to satisfy a specific performance index. Some typical examples are:

- Manipulability:

$$
f\left(\theta_{a}\right)=\sqrt{\operatorname{det}\left(\bar{J} \bar{J}^{T}\right)},
$$

which vanishes at a singular configuration;

- Distance from obstacles:

$$
f\left(\theta_{a}\right)=\min \left\|p\left(\theta_{a}\right)-p_{o}\right\|
$$

where $p_{o}$ is the position vector of a suitable point fixed on the obstacle and $p$ is the position vector of a generic point along the mechanism structure; 
- Joint limits: $\theta_{a m_{i}}<\theta_{a_{i}}<\theta_{a M_{i}}$,

$$
f\left(\theta_{a}\right)=-\frac{1}{2 n} \sum_{i=1}^{n}\left(\frac{\theta_{a_{i}}-\bar{\theta}_{a_{i}}}{\theta_{a M_{i}}-\theta_{a m_{i}}}\right)^{2},
$$

where $\theta_{a M_{i}}$ and $\theta_{a m_{i}}$ denote the maximum and minimum joint limits respectively, and $\bar{\theta}_{a_{i}}$ is the average value between $\theta_{a M_{i}}$ and $\theta_{a m_{i}}$.

\subsection{Kinematic singularities}

The posture of the manipulator, obtained as a function of the joint angles $\theta$, is said to be singular if the Jacobian matrix $\bar{J}$ has not full rank. From (8) we can observe that when the robot is not in a singular configuration it is possible to generate velocities and accelerations with the end-effector in certain directions. In order to evaluate the linear relation (8), the singular value decomposition (SVD) method can be used to obtain the rank of the Jacobian $\bar{J}$ and study quasi-linear mappings (Chiaverini et al., 2008).

In this context, the SVD of the Jacobian can be represented by

$$
\bar{J}=U \Sigma V^{T}=\sum_{i=1}^{m} \sigma_{i} u_{i} v_{i}^{T},
$$

where $U \in \mathbb{R}^{m \times m}$ is the orthogonal matrix of output singular vectors $u_{i}, V \in \mathbb{R}^{n \times n}$ is the orthogonal matrix of the input singular vectors $v_{i}$, and $\Sigma \in \operatorname{diag}(D, 0) \in$ $\mathbb{R}^{m \times n}$ is the matrix whose diagonal submatrix $D \in$ $\mathbb{R}^{m \times m}$ contains the singular values $\sigma_{i}$ of the matrix $\bar{J}$. Considering that $\operatorname{rank}(\bar{J})=k$, we have

- $\sigma_{1} \geq \sigma_{2} \geq \cdots \geq \sigma_{r} \geq \sigma_{k+1}=\ldots=0$;

- $\mathcal{R}(\bar{J})=\operatorname{span}\left\{u_{1}, \cdots, u_{k}\right\}$

- $\mathcal{N}(\bar{J})=\operatorname{span}\left\{v_{k+1}, \cdots, v_{n}\right\}$,

where $\mathcal{R}(\bar{J})$ denotes the range space of $\bar{J}$ and $\mathcal{N}(\bar{J})$ denotes the null space of $\bar{J}$. Then, the following analysis in terms of the rank of matrix $\bar{J}$ can be established:

- full $\operatorname{rank}(k=m):$ (i) $\sigma_{i} \neq 0, i=1, \cdots, m$, (ii) $\mathcal{R}(\bar{J}) \in$ $\mathbb{R}^{m},($ iii $) \mathcal{N}(\bar{J}) \in \mathbb{R}^{n-m}$.

- rank deficient $(k<m)$ : (i) $\sigma_{i} \neq 0, i=1, \cdots, k$, (ii) $\mathcal{R}(\bar{J}) \in \mathbb{R}^{k} \subset \mathbb{R}^{m}$, (iii) $\mathcal{N}(\bar{J}) \in \mathbb{R}^{n-k}$.

An interpretation of this analysis in terms of velocities is presented as follows (Chiaverini et al., 2008):
- Feasible velocities: For each configuration of the manipulator, $\mathcal{R}(\bar{J})$ is the set of the end-effector velocities that can be generated by all possible joint velocities $\dot{\theta}$, and are called the feasible velocities of the end-effector. The base of $\mathcal{R}(\bar{J})$ is obtained by the first $k$ output singular vectors, which represent the independent linear combinations of the single components of the endeffector velocities. Then, the effect of a singularity is to decrease the dimension of $\mathcal{R}(\bar{J})$, by eliminating a linear combination of the components of the endeffector velocities that belong to the feasible velocities space.

- Null space velocities: For each configuration of the manipulator, $\mathcal{N}(\bar{J})$ is the set of joint velocities $\dot{\theta}$, that do not produce any end-effector velocity, and are called the null space velocities. The base of $\mathcal{N}(\bar{J})$ is obtained by the last $n-k$ input singular vector, which represent the independent linear combinations of the velocities at each joint. The effect of a singularity is to increase the dimension of $\mathcal{N}(\bar{J})$, by adding more one independent linear combination of the joint velocities, which produces a zero end-effector velocity.

\section{NONHOLONOMIC ROBOTS}

Other examples of robotic systems with constraints are the wheeled mobile robots and multifingered robot hands. In general, a constraint is said to be holonomic if it restricts the robot motion to a smooth hypersurface of the configuration space. Holonomic constraints can be represented locally as algebraic constraints on the robot configuration space $c_{i}(\theta)=0$ for $i=1, \cdots, r$. On the other hand, if the allowable motions of the robotic system are restricted by the velocity constraint in the form

$$
A(\theta) \dot{\theta}=0,
$$

where $A \in \mathbb{R}^{r \times n}$ represents a set of $r$ velocity constraints that are not integrable, the constraint is said to be nonholonomic.

These constraints arise in robotic systems, where angular moment is conserved, as well as rolling contact is involved. Nonholonomic constraints occur when the instantaneous velocities of the robotic system are restricted to an $n-r$ dimensional subspace, but the set of reachable configurations is not constrained to some $n-r$ dimensional hypersurface in the robot configuration space (Murray et al., 1994).

Analogously, the challenge is how to consider the problem from the control point of view in order to define the system velocities which satisfy the constraints. Considering $\tilde{A} \in \mathbb{R}^{n \times(n-r)}$ the annihilator of matrix $A$, the 
allowable trajectories of the system can be written as possible solutions of the control system

$$
\dot{\theta}=\tilde{A}(\theta) u,
$$

where $u \in \mathbb{R}^{n-r}$ is the control signal to be designed in order to drive the current configuration $\theta \in \mathbb{R}^{n}$ to a desired time-varying configuration $\theta_{d}(t) \in \mathbb{R}^{n}$.

The main difficulty arises from the fact that this system has not right pseudoinverse, since $A$ contains more rows than columns, and the linearized system is not controllable (Murray et al., 1994). However, there are different approaches to accomplish the control of this systems, that consists of both physical remove of the nonholonomic constraints and the application of advanced control tools based on nonlinear control theory, differential geometry or optimal control (Murray et al., 1994; Bloch, 2003).

\section{SIMULATION RESULTS}

In this section, we present simulations results obtained from a Four-Bar linkage mechanism, a planar GoughStewart platform and two cooperating robot arms composed by four revolution joints. The adopted structural dimensions are: $l_{0}=1 \mathrm{~m}, l_{1}=1.2 \mathrm{~m}, l_{2}=1.4 \mathrm{~m}$, $l_{3}=0.6 \mathrm{~m}, l_{4}=1.4 \mathrm{~m}, l=5 \mathrm{~m}, l_{11}=l_{12}=1.2 \mathrm{~m}$, $l_{21}=l_{22}=1 \mathrm{~m}$ and $l_{13}=l_{23}=0.6 \mathrm{~m}$. For the considered lengths of links, we define the following limits: $\theta_{1} \in\left[\begin{array}{ll}0.72 & 2.27\end{array}\right] \mathrm{rad}$ and $\phi \in\left[\begin{array}{ll}5.88 & 7.89\end{array}\right] \mathrm{rad}$. The control parameters are: $k_{\phi}=50 \mathrm{rads}^{-1}$ and $K_{s}=K_{c}=$ $\operatorname{diag}\left(50 \mathrm{~mm} \mathrm{~s}^{-1}, 50 \mathrm{~mm} \mathrm{~s}^{-1}, 1 \mathrm{rads}^{-1}\right)$.

The time evolution for the orientation error of the FourBar linkage mechanism for train of ramps and sinusoidal references are shown in Figures 4(b) and 4(d) respectively. Figures 5(b) and 5(c) illustrate the time evolution of the position error and orientation error for the Gough-Stewart platform. The position and orientation errors for the object handled by two cooperating robots are shown in Figures 6(b) and 6(c) respectively. The trajectory following for all mechanisms are presented respectively in Figures 4(a)-4(c), 5(a) and 6(a), where it can observe that a good performance is achieved by using the presented methodology.

\section{CONCLUSIONS AND PERSPECTIVES}

This work presents a control methodology for robotic systems under kinematics constraints based on a novel scheme in the robotics literature, which regards the constraints on passive joint velocities. The key idea is to consider the kinematic constraints of the mechanism from their structure equations, rather than explicitly
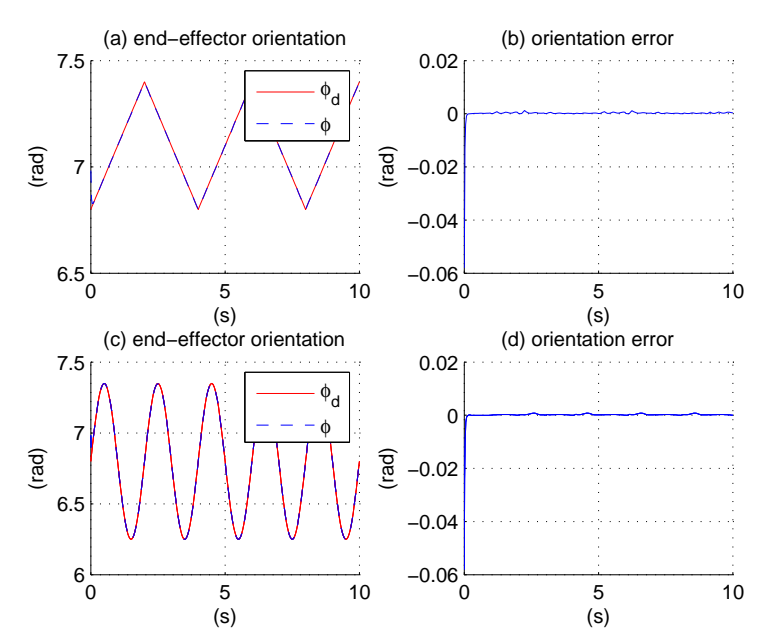

Figure 4: Four-Bar linkage mechanism: (a) end-effector orientation: train of ramps, (b) orientation error, (c) end-effector orientation: sinusoidal wave form, (d) orientation error.
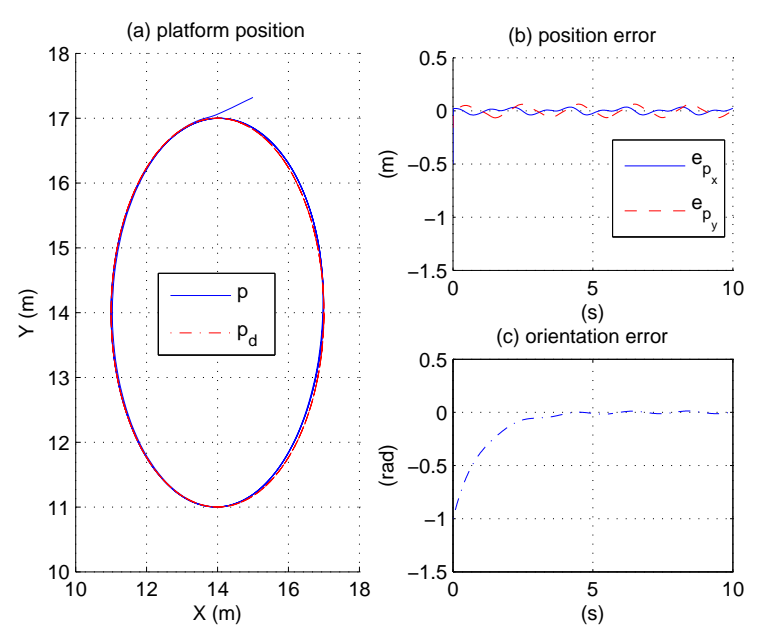

Figure 5: Gough-Stewart platform: (a) platform position, (b) position error, (c) orientation error.

invoking the constraint equation. In order to show the applicability of the presented methodology, simulations results were included for a Four-Bar linkage mechanism, a planar Gough-Stewart platform and two cooperating robots.

It is worth mentioning that the methodology presented in this section 3 is implemented in the suspension control system of the Environmental Hybrid Robot, recently developed by CENPES/Petrobras, which is an amphibious 4-wheel-legged robot composed by a planar parallel mechanism in each suspension (Freitas et al., 2010). 

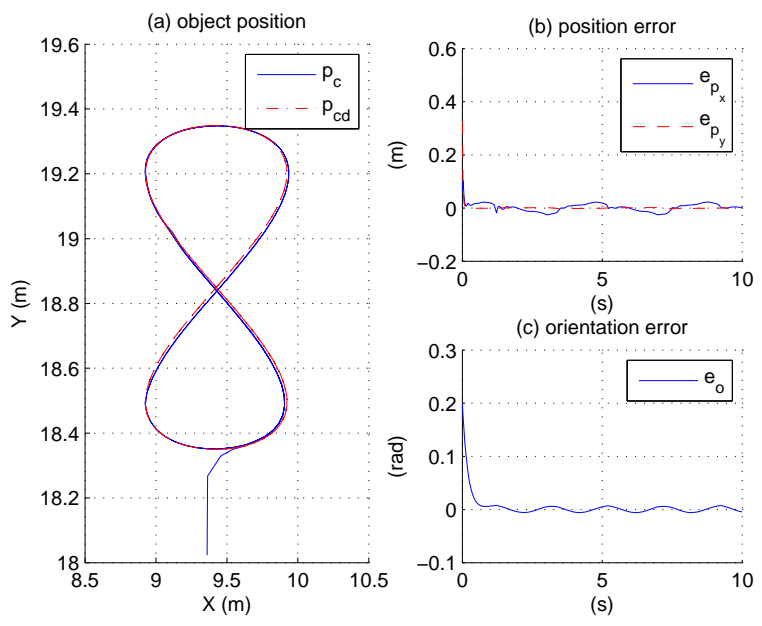

Figure 6: Cooperating robots: (a) object position, (b) position error, (c) orientation error.

Some research topics, applied to redundant manipulators and parallel robots, that can be investigated following the ideas presented in this work are: to consider the dynamic control problem for these mechanisms, relax the assumption of the robot kinematics to be fully known and develop a strategy for singularity and obstacle avoidance.

\section{ACKNOWLEDGMENTS}

This work was partially supported by Brazilian Founding agencies CNPq, CAPES and FAPERJ.

\section{REFERENCES}

Bicchi, A. and Prattichizzo, D. (2000). Manipulability of cooperating robots with unactuated joints and closed-chain mechanisms, IEEE Transactions on Robotics and Automation 16(4): 336-345.

Bloch, A. M. (2003). Nonholomic Mechanics and Control, Springer Verlag.

Caccavale, F. and Uchiyama, M. (2008). Cooperative manipulators, in B. Siciliano and O. Khatib (eds), Springer Handbook of Robotics, 1st edn, SpringerVerlag Ltd., pp. 701-718.

Caurin, G. A. P. and Pedro, L. M. (2009). Hybrid motion planning approach for robot dexterous hands, Journal of the Brazilian Society of Mechanical Sciences \& Engineering 31(4):289-296.

Cheng, H., Yiu, Y.-K. and Li, Z. (2003). Dynamics and control of redundantly actuated parallel manipula- tors, IEEE/ASME Transactions on Mechatronics 8(4): 483-491.

Chiaverini, S., Oriolo, G. and Walker, I. D. (2008). Kinematically redundant manipulators, in B. Siciliano and O. Khatib (eds), Springer Handbook of Robotics, 1st edn, Springer-Verlag Ltd., pp. 245268.

Freitas, G. M., Gleizer, G., Lizarralde, F., Hsu, L. and dos Reis, N. R. S. (2010). Kinematic reconfigurability control for an environmental mobile robot operating in the amazon rain forest, Journal of Field Robotics 27(2): 197-216.

Gosselin, C. and Angeles, J. (1990). Singularity analysis of closed-loop kinematic chains, IEEE Transactions on Robotics and Automation 6(3): 281-290.

Kim, J., Park, F. C., Ryu, S. J., Kim, J., Hwang, J. C., Park, C. and Iurascu, C. C. (2001). Design and analysis of a redundantly actuated parallel mechanism for rapid machining, IEEE Transactions on Robotics and Automation 17(4): 423-434.

Kövecses, J., Piedbœuf, J.-C. and Lange, C. (2003). Dynamics modeling and simulation of constrained robotic systems, IEEE/ASME Transactions on Mechatronics 8(2): 165-177.

Liu, G., Lou, Y. and Li, Z. (2003). Singularities of parallel manipulators: A geometric treatment, IEEE Transactions on Robotics and Automation 19(4): 579-594.

Merlet, J.-P. (1993). Parallel manipulators: state of the art and perspectives, Advanced Robotics 8(6): 589 596.

Merlet, J.-P. and Gosselin, C. (2008). Parallel mechanisms and robots, in B. Siciliano and O. Khatib (eds), Springer Handbook of Robotics, 1st edn, Springer-Verlag Ltd., pp. 269-285.

Murray, R. M., Li, Z. and Sastry, S. S. (1994). A Mathematical Introduction to Robotic Manipulation, 1st edn, CRC Press Inc., Boca Raton, FL, USA.

Namvar, M. and Aghili, F. (2005). Adaptive motionforce control of coordinated robots interacting with geometrically unknown environments, IEEE Transactions on Robotics 21(4): 678-694.

O'Brien, J. F., Jafari, F. and Wen, J. T. (2006). Determination of unstable singularities in parallel robots with $\mathrm{N}$-arms, IEEE Transactions on Robotics 22(1): 160-167. 
Pazelli, T., Terra, M. H. and Siqueira, A. A. (2011). Experimental investigation on adaptive robust controller designs applied to a free-floating space manipulator, Control Engineering Practice 1(1): 1-14.

Pietsch, I. T., Krefft, M., Becker, O. T., Bier, C. C. and Hesselbach, J. (2005). How to reach the dynamic limits of parallel robots? an autonomous control approach, IEEE Transactions on Automation Science and Engineering 2(4): 369-380.

Ribeiro, L., Guenther, R. and Martins, D. (2008). Screw-based relative jacobian for manipulators cooperating in a task, ABCM Symposium Series in Mechatronics 3: 276-285.

Rosario, J. M., Dumur, D. and Machado, J. T. A. (2007). Control of a 6-DOF parallel manipulator through a mechatronic approach, Journal of Vibration and Control 13(9): 1431-1446.

Sciavicco, L. and Siciliano, B. (2000). Modelling and Control of Robot Manipulators, 2nd edn, SpringerVerlag Ltd., New York, NY, USA.

Siciliano, B. (1990). Kinematic control of redundant robot manipulators: A tutorial, Journal of Intelligent and Robotic Systems 3(3): 201-212.

Siciliano, B., Sciavicco, L., Villani, L. and Oriolo, G. (2008). Robotics: Modelling, Planning and Control, 1st edn, Springer-Verlag London Ltd.

Simas, H., Guenther, R., da Cruz, D. F. M. and Martins, D. (2009). A new method to solve robot inverse kinematics using Assur virtual chains, Robotica 27: 1017-1022.

Tinos, R., Terra, M. H. and Ishihara, J. Y. (2006). Motion and force control of cooperative robotic manipulators with passive joints, IEEE Transactions on Control System Technology 14(4): 725-734.

Wen, J. T. and O'Brien, J. F. (2003). Singularities in three-legged platform-type parallel mechanisms, IEEE Transactions on Robotics and Automation 19(4): 720-727.

Wen, J. T.-Y. and Wilfinger, L. S. (1999). Kinematic manipulability of general constrained rigid multibody systems, IEEE Transactions on Robotics and Automation 15(3): 558-567.

Zhang, G.-F. and Gao, X.-S. (2006). Planar generalized stewart platforms and their direct kinematics, in H. Hong and D. Wang (eds), Automated Deduction in Geometry, Vol. 3763 of Lecture Notes in Computer Science, Springer-Verlag Berlin / Heidelberg, pp. 198-211. 